Arch. Math. 76 (2001) 41-50

0003-889X/01/010041-10 \$3.50/0

(C) Birkhäuser Verlag, Basel, 2001

Archiv der Mathematik

\title{
The pair correlation of zeros of the Riemann zeta function and distribution of primes
}

\author{
By \\ JIANYA LIU ${ }^{1}$ ) and YANGBO YE$^{2}$ )
}

\begin{abstract}
Assuming a special version of the Montgomery-Odlyzko law on the pair correlation of zeros of the Riemann zeta function conjectured by Rudnick and Sarnak and assuming the Riemann Hypothesis, we prove new results on the prime number theorem, difference of consecutive primes, and the twin prime conjecture.
\end{abstract}

1. Introduction. Assuming the Riemann Hypothesis (RH), let us denote by $\rho=1 / 2+i \gamma$ a nontrivial zero of a primitive $L$-function $L(s, \pi)$ attached to an irreducible cuspidal automorphic representation of $\mathrm{GL}_{m}, m \geqq 1$, over $\mathbb{Q}$. When $m=1$, this $L$-function is the Riemann zeta function $\zeta(s)$ or the Dirichlet $L$-function $L(s, \chi)$ for a primitive character $\chi$. Rudnick and Sarnak [13] examined the $n$-level correlation for these zeros and made a far reaching conjecture which is called the Montgomery [9]-Odlyzko [11], [12] Law by Katz and Sarnak [6]. Rudnick and Sarnak also proved a case of their conjecture when a test function $f$ has its Fourier transform $\widehat{f}$ supported in a restricted region.

In this article, we will show that a version of the above conjecture for the pair correlation of zeros of the zeta function $\zeta(s)$ implies interesting arithmetical results on prime distribution (Theorems 2, 3, and 4). These results can give us deep insight on possible ultimate bounds of these prime distribution problems. One can also see that the pair (and $n$ level) correlation of zeros of zeta and $L$-functions is a powerful method in number theory. Our computation shows that the test function $f$ and the support of its Fourier transform $\widehat{f}$ play a crucial role in the conjecture.

To see the conjecture in Rudnick and Sarnak [13] in the case of the zeta function $\zeta(s)$ and $n=2$, the pair correlation, we use a test function $f(x, y)$ which satisfies the following three conditions:

(i) $f(x, y)=f(y, x)$ for any $x, y \in \mathbb{R}$,

(ii) $f(x+t, y+t)=f(x, y)$ for any $t \in \mathbb{R}$, and

(iii) $f(x, y)$ tends to 0 rapidly as $|(x, y)| \rightarrow \infty$ on the hyperplane $x+y=0$.

Mathematics Subject Classification (1991): 11M26, 11N05, 11 N75.

1) Supported in part by China NNSF Grant \# 19701019.

$\left.{ }^{2}\right)$ Supported in part by USA NSF Grant \# DMS 97-01225. 
Define the function

$$
W_{2}(x, y)=1-\frac{\sin ^{2} \pi(x-y)}{(\pi(x-y))^{2}} .
$$

Denote the Dirac function by $\delta(x)$ which satisfies $\int_{\mathbb{R}} \delta(x) d x=1$ and defines a distribution $f \mapsto f(0)$. We then define the pair correlation sum of zeros $\gamma_{j}$ of the zeta function:

$$
R_{2}(T, f, h)=\sum_{\substack{\gamma_{1}, \gamma_{2} \\ \text { distinct }}} h\left(\frac{\gamma_{1}}{T}, \frac{\gamma_{2}}{T}\right) f\left(\frac{L \gamma_{1}}{2 \pi}, \frac{L \gamma_{2}}{2 \pi}\right)
$$

where $T \geqq 2, L=\log T$, and $h(x, y)$ is a localized cutoff function which tends to zero rapidly when $|(x, y)|$ tends to infinity. The conjecture proposed by Rudnick and Sarnak [13] is that

$$
R_{2}(T, f, h) \sim \frac{1}{2 \pi} T L \int_{\mathbb{R}} h(r, r) d r \int_{\mathbb{R}^{2}} f(x, y) W_{2}(x, y) \delta\left(\frac{x+y}{2}\right) d x d y
$$

holds universally for any function $f$ satisfying the three conditions, and for a sufficiently rich family of localized cutoff functions $h$.

Now we turn to the version of Montgomery-Odlyzko Law which we will use in this paper. Denote $w(u)=4 /\left(4+u^{2}\right)$ and define $h\left(r_{1}, r_{2}\right)$ to be the characteristic function of $\left[L^{-4}, 1\right] \times\left[L^{-4}, 1\right]$. Our goal is to estimate the sum

$$
\sum_{T L^{-4} \leqq \gamma_{1}, \gamma_{2} \leqq T} w\left(\gamma_{1}-\gamma_{2}\right) e\left(\xi\left(\gamma_{1}-\gamma_{2}\right)\right),
$$

where $e(x)=e^{2 \pi i x}$. Note that the terms with $\gamma_{1}=\gamma_{2}$ contribute the sum $\sum_{T L^{-4} \leqq \gamma \leqq T} 1$. Using the function $h\left(r_{1}, r_{2}\right)$ above, we can find a function $f_{T}$ on $\mathbb{R}^{2}$ satisfying the three conditions such that

$$
\begin{aligned}
R_{2}\left(T, f_{T}, h\right) & =\sum_{T L^{-4} \leqq \gamma_{1}, \gamma_{2} \leqq T}^{\substack{\text { distinct }\\
}} f_{T}\left(\frac{L \gamma_{1}}{2 \pi}, \frac{L \gamma_{2}}{2 \pi}\right) \\
& \sum_{\substack { T L^{-4} \\
\begin{subarray}{c}{\text { distinct } \\
\text { dinct }{ T L ^ { - 4 } \\
\begin{subarray} { c } { \text { distinct } \\
\text { dinct } } }\end{subarray}} w\left(\gamma_{1}-\gamma_{2}\right) e\left(\xi\left(\gamma_{1}-\gamma_{2}\right)\right) .
\end{aligned}
$$

More precisely, we can take

$$
f_{T}(x,-x)= \begin{cases}w(4 \pi x / L) \cos \left(8 \pi^{2} \xi x / L\right) & \text { if }|x| \leqq T L /(4 \pi) \\ 0 & \text { otherwise }\end{cases}
$$

on the line $x+y=0$ and then extend its definition to $\mathbb{R}^{2}$ by $f_{T}((x, y)+(t, t))=f_{T}(x, y)$, $x, y \in \mathbb{R}$. Here $\xi$ is a positive parameter. Note that this function satisfies the three conditions. We will assume the following conjecture in this article.

Conjecture 1. With the above notation and functions $f_{T}$ and $h$, we have

$$
R_{2}\left(T, f_{T}, h\right)=\frac{1}{2 \pi} T L \int_{\mathbb{R}^{2}} f_{T}(x, y) W_{2}(x, y) \delta\left(\frac{x+y}{2}\right) d x d y+O(T)
$$

where the constant in $O$ is independent of $\xi$. 
We note that for the function $h$ chosen above, we have $\int_{\mathbb{R}} h(r, r) d r=\int_{L^{-4}}^{1} d r=1-L^{-4}$. As pointed out in [13], our function $h$ localizes $\gamma$ to be of order $T$, and the normalization $\gamma L /(2 \pi)$ in $R_{2}\left(T, f_{T}, h\right)$ is the same as $\tilde{\gamma}=\gamma \log |\gamma| /(2 \pi)$.

From Theorem 1 and its Corollary below we can see that Conjecture 1 implies the original Montgomery Conjecture [9]. The strength of Conjecture 1, as a special version of the Montgomery-Odlyzko law conjectured by Rudnick and Sarnak [13], lies in the remainder term $O(T)$ in (3). When the test function $f_{T}$ is independent of $T$ and has a restricted support of its Fourier transform, this remainder term was indeed proved in [13], p. 284, taken into account that the number of nontrivial zeros with $0 \leqq \gamma \leqq T$ is $(1 / 2 \pi) T L+O(T)$. We also want to point out that the fact that our test function $f_{T}$ depends on $T$ can be obtained by requiring the conjecture in (1) to hold uniformly for $f$ in a certain sense.

The authors would like to thank the referee for helpful suggestions in detail.

2. The main results. We will compute the integral on the right side of (3) and prove the following theorem in Section 3.

Theorem 1. For $T \geqq 2$ and $\xi>0$ denote

$$
F(T, \xi)=\sum_{T L^{-4}<\gamma_{1}, \gamma_{2} \leqq T} w\left(\gamma_{1}-\gamma_{2}\right) e\left(\xi\left(\gamma_{1}-\gamma_{2}\right)\right)
$$

where $\gamma_{1}$ and $\gamma_{2}$ run through the imaginary part of the non-trivial zeros $\rho=1 / 2+i \gamma$ of $\zeta(s)$ counting multiplicities. Then under the Riemann Hypothesis and Conjecture 1 we have

$$
F(T, \xi)=\frac{T L}{2 \pi} \min \left(1, \frac{2 \pi \xi}{L}\right)+\frac{T L^{2}}{2 \pi} e^{-4 \pi \xi}+O(T)
$$

uniformly for $\xi$.

Corollary. For $T \geqq 2$ and $\xi>0$ we set

$$
G(T, \xi)=\sum_{0<\gamma_{1}, \gamma_{2} \leqq T} w\left(\gamma_{1}-\gamma_{2}\right) e\left(\xi\left(\gamma_{1}-\gamma_{2}\right)\right) \text {. }
$$

Then, assuming RH and Conjecture 1, we have

$$
G(T, \xi)=\frac{T L}{2 \pi} \min \left(1, \frac{2 \pi \xi}{L}\right)+\frac{T L^{2}}{2 \pi} e^{-4 \pi \xi}+O(T)
$$

uniformly for $\xi$.

In Section 4 we will estimate $S(T, 0, \xi)$ based on this corollary, where

$$
S(T, v, \xi)=\sum_{0<\gamma \leqq T} e(\gamma(v+\xi)) .
$$

Recall the following classical results under RH by von Koch [7] and Cramér [1]

$$
\begin{aligned}
& \psi(x)=x+O\left(x^{\frac{1}{2}} \log ^{2} x\right), \\
& p_{n+1}-p_{n} \ll p_{n}^{\frac{1}{2}} \log p_{n} .
\end{aligned}
$$


Here, as usual, $\psi(x)$ is the sum of the von Mongoldt function $\Lambda(n)$ up to $n \leqq x$, and $p_{n}$ is the $n$-th prime. By assuming Montgomery's pair correlation conjecture in addition to $\mathrm{RH}$, Gallagher and Mueller [3] improved the prime number theorem to the form

$$
\psi(x)=x+o\left(x^{\frac{1}{2}} \log ^{2} x\right)
$$

and Mueller [10] showed that

$$
p_{n+1}-p_{n} \ll p_{n}^{\frac{1}{2}} \log ^{\frac{3}{4}+\varepsilon} x
$$

for any $\varepsilon>0$. Under the same assumptions, the last result was further improved by HeathBrown [4] and Heath-Brown and Goldston [5]:

$$
p_{n+1}-p_{n}=o\left(p_{n}^{\frac{1}{2}} \log ^{\frac{1}{2}} p_{n}\right) \text {. }
$$

In Section 5, we will prove the following theorems in this direction.

Theorem 2. Assume RH and Conjecture 1. Then

$$
\psi(x)=x+O\left(x^{\frac{1}{2}} \log ^{\frac{5}{4}} x\right) .
$$

Here we remark that our Theorem 2 is closer to the best possible bound, in view of the $\Omega$-result

$$
\psi(x)-x=\Omega\left(x^{\frac{1}{2}} \log \log \log x\right)
$$

of Littlewood [8].

Theorem 3. Assume $R H$ and Conjecture 1. Then

$$
p_{n+1}-p_{n} \ll p_{n}^{\frac{1}{2}} \log ^{\frac{1}{4}} p_{n} .
$$

A classical conjecture states that there is always a prime between two consecutive squares. One easily sees that Theorem 3 is weaker than this conjecture by a factor of $\log ^{\frac{1}{4}} p_{n}$.

The twin prime conjecture states that $\liminf _{n \rightarrow \infty}\left(p_{n+1}-p_{n}\right)=2$. Assuming Montgomery's pair correlation conjecture and RH, Heath-Brown [4] showed that

$$
\liminf _{n \rightarrow \infty} \frac{p_{n+1}-p_{n}}{\log p_{n}}=0 .
$$

We will prove the following theorem.

Theorem 4. Assume RH and Conjecture 1. We have

$$
\liminf _{n \rightarrow \infty} \frac{p_{n+1}-p_{n}}{\log ^{\frac{2}{3}} p_{n}}<+\infty .
$$

3. Estimation of $\boldsymbol{F}(\boldsymbol{T}, \boldsymbol{\xi})$ and $\boldsymbol{G}(\boldsymbol{T}, \boldsymbol{\xi})$. In this section we first compute the integral

$$
\int_{\mathbb{R}^{2}} f_{T}(x, y) W_{2}(x, y) \delta\left(\frac{x+y}{2}\right) d x d y .
$$

Recall that $\delta(x)$ is the Dirac mass at zero. Changing variables $u=x-y$ and $t=(x+y) / 2$, 
we get $f_{T}(x, y)=f_{T}(x-t, y-t)=f_{T}(u / 2,-u / 2)$. Note that $W_{2}(x, y)=1-\sin ^{2} \pi u /(\pi u)^{2}$.

Hence

$$
\begin{aligned}
\int_{\mathbb{R}^{2}} f_{T}(x, y) W_{2}(x, y) \delta\left(\frac{x+y}{2}\right) d x d y \\
=\int_{-T L /(2 \pi)}^{T L /(2 \pi)} w\left(\frac{2 \pi u}{L}\right) e\left(\frac{2 \pi \xi u}{L}\right)\left(1-\left(\frac{\sin \pi u}{\pi u}\right)^{2}\right) d u \int_{\mathbb{R}} \delta(t) d t \\
=\frac{1}{\pi} \int_{-T L / 2}^{T L / 2} w\left(\frac{2 x}{L}\right) e\left(\frac{2 \xi x}{L}\right)\left(1-\left(\frac{\sin x}{x}\right)^{2}\right) d x \\
=\frac{1}{\pi} \int_{\mathbb{R}} e^{4 \pi i \xi x / L} \frac{d x}{1+(x / L)^{2}}-\frac{1}{\pi} \int_{\mathbb{R}} e^{4 \pi i \xi x / L} \cdot \frac{\sin ^{2} x}{x^{2}\left(1+(x / L)^{2}\right)} d x+O\left(\frac{L}{T}\right) .
\end{aligned}
$$

By

$$
\frac{1}{x^{2}\left(1+(x / L)^{2}\right)}=\frac{1}{x^{2}}-\frac{1}{L^{2}+x^{2}}
$$

we get

$$
\begin{aligned}
& \int_{\mathbb{R}^{2}} f_{T}(x, y) W_{2}(x, y) \delta\left(\frac{x+y}{2}\right) d x d y \\
& =\min \left(1, \frac{2 \pi \xi}{L}\right)-1+\left(L+\frac{1}{2 L}\right) e^{-4 \pi \xi}-\frac{1}{4 L} e^{-(4 \pi \xi+2 L)}-\frac{1}{4 L} e^{-|4 \pi \xi-2 L|}+O\left(\frac{L}{T}\right) .
\end{aligned}
$$

Consequently the conjectured asymptotic formula in (3) implies that

Recall that

$$
R_{2}\left(T, f_{T}, h\right)=\frac{T L}{2 \pi} \min \left(1, \frac{2 \pi \xi}{L}\right)-\frac{T L}{2 \pi}+\frac{T L^{2}}{2 \pi} e^{-4 \pi \xi}+O(T)
$$

$$
\begin{aligned}
R_{2}\left(T, f_{T}, h\right) & =\sum_{T / L^{4} \leqq \gamma_{1}, \gamma_{2} \leqq T} w\left(\gamma_{1}-\gamma_{2}\right) e\left(\xi\left(\gamma_{1}-\gamma_{2}\right)\right)-\sum_{T / L^{4} \leqq \gamma \leqq T} 1 \\
& =\sum_{T / L^{4} \leqq \gamma_{1}, \gamma_{2} \leqq T} w\left(\gamma_{1}-\gamma_{2}\right) e\left(\xi\left(\gamma_{1}-\gamma_{2}\right)\right)-\frac{T L}{2 \pi}+O(T) .
\end{aligned}
$$

We therefore get a proof of Theorem 1 .

To prove the Corollary, we observe that

$$
G(T, \xi)-F(T, \xi) \ll\left|G\left(T L^{-4}, \xi\right)\right|+|Q|,
$$

where

$$
Q=\sum_{\substack{0<\gamma_{1} \leqq T L^{-4} \\ 0<\gamma_{2} \leqq T}} w\left(\gamma_{1}-\gamma_{2}\right) e\left(\xi\left(\gamma_{1}-\gamma_{2}\right)\right) .
$$

To estimate $Q$, we let $k(u)=2 \pi \exp (-4 \pi|v|)$, and set

$$
S(T, v, \xi)=\sum_{0<\gamma \leqq T} e(\gamma(v+\xi)) .
$$

Then we have $\int_{\mathbb{R}} k(v) e(v x) d v=w(x)$ and consequently $\int_{\mathbb{R}} k(v)|S(T, v, \xi)|^{2} d v=G(T, \xi)$. 
Applying the above two formulae to $Q$, we get

$$
\begin{aligned}
Q= & \int_{-\infty}^{+\infty} k(v) S\left(T L^{-4}, v, \xi\right) \overline{S(T, v, \xi)} d v \\
& \ll\left(\int_{-\infty}^{+\infty} k(v)\left|S\left(T L^{-4}, v, \xi\right)\right|^{2} d v\right)^{\frac{1}{2}}\left(\int_{-\infty}^{+\infty} k(v)|S(T, v, \xi)|^{2} d v\right)^{\frac{1}{2}} \\
& \ll\left|G\left(T L^{-4}, \xi\right)\right|^{\frac{1}{2}}|G(T, \xi)|^{\frac{1}{2}} .
\end{aligned}
$$

Inserting this into (6) and then using the trivial bound $G(t, \xi) \ll t \log ^{2} t$, which holds for all $t$ and $\xi$, we get

$$
G(T, \xi)-F(T, \xi) \ll T L^{-2}+\left(T L^{-2}\right)^{\frac{1}{2}}\left(T L^{2}\right)^{\frac{1}{2}} \ll T .
$$

Then the corollary is a consequence of this result and (4).

4. Estimation of $\boldsymbol{S}(\boldsymbol{T}, \mathbf{0}, \boldsymbol{\xi})$. In this section we give an estimate of the exponential sum $S(T, \xi)=S(T, 0, \xi)$ defined as in (7). Let

$$
G_{\beta}(T, \xi)=\sum_{0<\gamma_{1}, \gamma_{2} \leqq T} w\left(\frac{\gamma_{1}-\gamma_{2}}{\beta}\right) e\left(\xi\left(\gamma_{1}-\gamma_{2}\right)\right) .
$$

Obviously $G_{1}(T, \xi)=G(T, \xi)$. We bound $S(T, \xi)$ via $G_{\beta}(T, \xi)$ rather than appeal to the estimate for $G(T, \xi)$ directly. This approach, used by Heath-Brown and Goldston [5] for the first time, can give us extra saving on the upper bound of $S(T, \xi)$. The first two lemmas below are Lemmas 1 and 2 in [5]; note that our $\xi$ and their $x$ are related by $\xi=\log x /(2 \pi)$.

Lemma 1. We have

$$
S(T, \xi) \ll\left(\frac{T}{\beta} \max _{t \leqq T} G_{\beta}(t, \xi)\right)^{\frac{1}{2}}
$$

uniformly for all $0<\beta \leqq T$ and $\xi>0$.

Lemma 2. For $\beta>0, t \geqq 2$ and $\xi>0$, we have

$$
G_{\beta}(t, \xi)=\beta^{2} G(t, \xi)+\beta\left(1-\beta^{2}\right) \int_{\mathbb{R}} G(t, u) \min \left\{e^{2 \beta(u-\xi)}, e^{2 \beta(\xi-u)}\right\} d u .
$$

Lemma 3. Assume $R H$ and the Conjecture 1. Then uniformly for $\log T \ll \xi$, we have

$$
S(T, \xi) \ll T \log ^{\frac{1}{4}} T .
$$

Proof of Lemma 3. We write

$$
\int_{\mathbb{R}} G(t, u) \min \left\{e^{2 \beta(u-\xi)}, e^{2 \beta(\xi-u)}\right\} d u=\int_{-\infty}^{\frac{\xi}{2}}+\int_{\frac{\xi}{2}}^{+\infty},
$$


and estimate the last two integrals separately. First

$$
\int_{-\infty}^{\frac{\xi}{2}} \ll t \log ^{2} t \int_{-\infty}^{\frac{\xi}{2}} e^{2 \beta(u-\xi)} d u \ll \frac{t \log ^{2} t}{\beta} e^{-\beta \xi}
$$

by $G(t, u)=G(t,-u)$ and the trivial estimate $G(t, \xi) \ll t \log ^{2} t$. By the corollary, we have

$$
\begin{aligned}
\int_{\xi / 2}^{+\infty} & =\int_{\xi / 2}^{+\infty}\left(\frac{t \log t}{2 \pi}+O(t)\right) \min \left\{e^{2 \beta(u-\xi)}, e^{2 \beta(\xi-u)}\right\} d u \\
& =\left(\frac{t \log t}{2 \pi}+O(t)\right)\left(\int_{\frac{\xi}{2}}^{\xi} e^{2 \beta(u-\xi)} d u+\int_{\xi}^{+\infty} e^{2 \beta(\xi-u)} d u\right) \\
& =\frac{t \log t}{2 \pi \beta}+O\left(\frac{t}{\beta}\right)+O\left(\frac{t \log t}{\beta} e^{-\beta \xi}\right) .
\end{aligned}
$$

Therefore

$$
\int_{\mathbb{R}} G(t, u) \min \left\{e^{2 \beta(u-\xi)}, e^{2 \beta(\xi-u)}\right\} d u=\frac{t \log t}{2 \pi \beta}+O\left(\frac{t}{\beta}\right)+O\left(\frac{t \log ^{2} t}{\beta} e^{-\beta \xi}\right) .
$$

Now we apply the above estimate and the corollary to the formula in Lemma 2 . Then, uniformly for $t \leqq T$, we get

$$
\begin{aligned}
G_{\beta}(t, u) & =\beta^{2} G(t, \xi)+\beta\left(1-\beta^{2}\right)\left\{\frac{t \log t}{2 \pi \beta}+O\left(\frac{t}{\beta}\right)+O\left(\frac{t \log ^{2} t}{\beta} e^{-\beta \xi}\right)\right\} \\
& =\frac{t \log t}{2 \pi}+O\left(\beta^{2} t\right)+O\left(\beta^{2} e^{-\beta \xi} t \log ^{2} t\right) .
\end{aligned}
$$

Inserting this into Lemma 1, we obtain

$$
S(T, \xi) \ll \frac{T \log ^{\frac{1}{2}} T}{\beta^{\frac{1}{2}}}+\beta^{\frac{1}{2}} T+\beta^{\frac{1}{2}} e^{-\frac{\beta \xi}{2}} T \log T,
$$

which is $\ll T \log ^{\frac{1}{4}} T$, by choosing $\beta=\log ^{\frac{1}{2}} T$ and using $\xi \gg \log T$.

\section{Distribution of primes.}

Lemma 4. Assume RH. Then for $x, T \geqq 2$ we have

$$
\psi(x)=x-\sum_{|\gamma| \leqq T} \frac{x^{\frac{1}{2}+i \gamma}}{\frac{1}{2}+i \gamma}+E(x)+F(x),
$$

where $E(x)=E(x, T) \ll 1 / x^{2}+x(\log x T)^{2} / T$, and $F(x)=F(x, T) \ll \Lambda(n)$ for $n-\frac{1}{2}<x \leqq$ $n+\frac{1}{2}$.

This follows from Davenport [2], Chapter 17. See also Heath-Brown [4], Lemma 1.

Proof of Theorem 2. Taking $T=x$ in Lemma 4, we get by partial summation that

$$
\psi(x)-x \ll x^{\frac{1}{2}}\left\{1+\frac{|S(x, \xi)|}{x}+\int_{2}^{x} \frac{|S(t, \xi)|}{t^{2}} d t\right\}
$$


where $\xi=\log x /(2 \pi)$. For $2 \leqq t \leqq x$, we trivially have $\log t \ll \xi$, so Lemma 3 gives us

$$
\psi(x)-x \ll x^{\frac{1}{2}}\left\{1+\log ^{\frac{1}{4}} x+\int_{2}^{x} \frac{\log ^{\frac{1}{4}} t}{t} d t\right\} \ll x^{\frac{1}{2}} \log ^{\frac{5}{4}} x .
$$

This is the desired result.

Proof of Theorem 3. Let $1<h<x$. For $x \leqq y \leqq x+h$, we get by Lemma 4 that

$$
\psi(y+h)-\psi(y)-h=-\sum_{|\gamma| \leqq x} \frac{(y+h)^{\rho}-y^{\rho}}{\rho}+O\left(\log ^{2} x\right),
$$

where $\rho=\frac{1}{2}+i \gamma$ is a non-trivial zero of the zeta-function. Therefore,

$$
\begin{aligned}
\int_{x}^{x+h}(\psi(y+h) & -\psi(y)) d y-h^{2}=-\int_{x}^{x+h} \sum_{|\gamma| \leqq x / h} \frac{(y+h)^{\rho}-y^{\rho}}{\rho} d y \\
& -\int_{x}^{x+h} \sum_{x / h<|\gamma| \leqq x} \frac{(y+h)^{\rho}-y^{\rho}}{\rho} d y+O\left(h \log ^{2} x\right) .
\end{aligned}
$$

Denote by $I_{1}$ the first integral on the right side and by $I_{2}$ the second integral. The integral $I_{1}$ can be written as

$$
I_{1}=\int_{x}^{x+h} \int_{y}^{y+h}\left(\sum_{|\gamma| \leqq x / h} z^{\rho-1}\right) d z d y=\int_{x}^{x+h} \int_{y}^{y+h} z^{-\frac{1}{2}} S\left(\frac{x}{h}, \xi\right) d z d y
$$

with $\xi=\log z /(2 \pi)$. Clearly $\log (x / h) \ll \log \xi$, so Lemma 3 implies that

$$
I_{1} \ll \frac{h^{2}}{x^{\frac{1}{2}}}\left(\frac{x}{h}\right)\left(\log \frac{x}{h}\right)^{\frac{1}{4}} \ll h x^{\frac{1}{2}} \log ^{\frac{1}{4}} x .
$$

To estimate $I_{2}$, we note that

$$
I_{2}=\sum_{x / h<|\gamma| \leqq x} \frac{(x+2 h)^{\rho+1}-2(x+h)^{\rho+1}+x^{\rho+1}}{\rho(\rho+1)} .
$$

By partial summation and Lemma 3 with $\xi=\log x /(2 \pi)$, we have

$$
\begin{aligned}
\sum_{x / h<|\gamma| \leqq x} \frac{x^{\rho+1}}{\rho(\rho+1)} & \ll x^{\frac{3}{2}}\left\{\frac{|S(x, \xi)|}{x^{2}}+\frac{|S(x / h, \xi)|}{(x / h)^{2}}+\int_{x / h}^{x} \frac{|S(t, \xi)|}{t^{3}} d t\right\} \\
& \ll x^{\frac{3}{2}}\left\{\frac{\log ^{\frac{1}{4}} x}{x}+\frac{h \log ^{\frac{1}{4}} x}{x}+\int_{x / h}^{x} \frac{\log ^{\frac{1}{4}} t}{t^{2}} d t\right\} \ll h x^{\frac{1}{2}} \log ^{\frac{1}{4}} x .
\end{aligned}
$$

The sums

$$
\sum_{x / h<|\gamma| \leqq x} \frac{(x+2 h)^{\rho+1}}{\rho(\rho+1)}, \sum_{x / h<|\gamma| \leqq x} \frac{(x+h)^{\rho+1}}{\rho(\rho+1)}
$$


can be estimated by the same method. Thus $I_{2} \ll h x^{\frac{1}{2}} \log ^{\frac{1}{4}} x$. Inserting this and (9) into (8), we get

$$
\int_{x}^{x+h}(\psi(y+h)-\psi(y)) d y=h^{2}+O\left(h x^{\frac{1}{2}} \log ^{\frac{1}{4}} x\right),
$$

where the $O$-constant is absolute. Let $h=C x^{\frac{1}{2}} \log ^{\frac{1}{4}} x$, where $C$ is an absolute constant such that $h^{2}+O\left(h x^{\frac{1}{2}} \log ^{\frac{1}{4}} x\right) \geqq h^{2} / 2$. Then (10) implies that there exists $y_{0}$ with $x \leqq y_{0} \leqq x+h$ such that $\psi\left(y_{0}+h\right)-\psi\left(y_{0}\right) \geqq h / 2$. Therefore $\psi(x+2 h)-\psi(x) \geqq \psi\left(y_{0}+h\right)-\psi\left(y_{0}\right) \geqq h / 2$, because $\psi(t)$ is non-decreasing in $t$. This shows that there is a prime in every interval $[x, x+2 h]$, from which the theorem follows.

Before proving Theorem 4, we need a lemma which is Theorem 3 in Heath-Brown [5].

Lemma 5. Assume $R H$ and set $\xi=\log x /(2 \pi)$. Suppose

$$
|G(T, \xi)-T \xi| \leqq \delta(x) T \log T
$$

for some function $\delta(x)$ with $\log ^{-\frac{1}{3}} x \leqq \delta(x) \leqq 1$ and for $x \log ^{-2} x \leqq T \leqq x \log x$. Then there exist numerical constants $c_{1}, c_{2}$ such that

$$
\min _{x / c_{1}<p_{n} \leqq c_{1} x} \frac{p_{n+1}-p_{n}}{\log p_{n}} \leqq c_{2} \delta(x) .
$$

Proof of The orem 4. By the corollary, the conjecture in (3) implies for $\log T \ll \xi$ that

$$
G(T, \xi)=\frac{T \log T}{2 \pi} \min \left(1, \frac{2 \pi \xi}{\log T}\right)+O(T) .
$$

If $x \log { }^{-2} x \leqq T \leqq x$, then $\frac{2 \pi \xi}{\log T} \geqq 1$ and $\log T=\log x+O(\log \log x)$. Hence in this case (12) becomes $G(T, \xi)-T \xi \ll T \log \log x$. It is easy to check that the above estimates is still true when $x \leqq T \leqq x \log x$, thus (11) holds with $\delta(x)=c_{3} \log ^{-\frac{1}{3}} x$, where $c_{3} \geqq 1$ is a numerical constant. Theorem 4 now follows from Lemma 5 .

\section{References}

[1] H. CRAmÉr, Some theorems concerning prime numbers. Arkiv Mat. Astronomy Fysik 15, 1-32 (1920).

[2] H. Davenport, Multiplicative number theory. Chicago 1967.

[3] P. X. Gallagher and J. H. Mueller, Primes and zeros in short intervals. J. Reine Angew. Math. 303/304, $205-220$ (1978).

[4] D. R. Heath-Brown, Gaps between primes, and the pair correlation of zeros of the zeta-function. Acta Arith. 41, 85-99 (1982).

[5] D. R. Heath-Brown and D. A. Goldston, A note on the differences between consecutive primes. Math. Ann. 266, 317-320 (1984).

[6] N. M. KatZ and P. SARnaK, Random Matrices, Frobenius Eigenvalues, and Monodromy. Colloq. Publ. 45, Amer. Math. Soc., Providence, 1999.

[7] H. vON Koch, Sur la distribution des nombres premiers. Acta Math. 24, 159-182 (1901).

[8] J. E. LitTlewood, Sur la distribution des nombres premiers. C.R. Hebd. Séanc. Acad. Sci. Paris 158, $1869-1872$ (1914).

[9] H. L. Montgomery, The pair correlation of zeros of the zeta function. Proc. Symp. Pure Math. 24, 181 -193, Amer. Math. Soc., Providence, 1973. 
[10] J. H. Mueller, On the difference between consecutive primes. In: Recent Progress in Analytic Number Theory I, 269-273. Durham 1981.

[11] A. M. OdLyzko, On the distribution of spacings between zeros of the zeta function. Math. Comp. 48, $273-308$ (1987).

[12] A. M. OdLyzko, The $10^{20}$ zero of the Riemann zeta function and 70 million of its neighbors. Preprint 1989.

[13] Z. RUDNICK and P. SARNAK, Zeros of principal $L$-functions and random matrix theory. Duke Math. J. 81, 269-322 (1996).

Eingegangen am 4. 6. 1999

Anschrift der Autoren:

Jianya Liu

Department of Mathematics

Shandong University

Jinan 250100

China

Department of Mathematics

The University of Hong Kong

Hong Kong

\author{
Yangbo Ye \\ Department of Mathematics \\ The University of Iowa \\ Iowa City, IA 52242 \\ USA \\ Department of Mathematics \\ The University of Hong Kong \\ Hong Kong \\ Department of Mathematics \\ Shandong University \\ Jinan 250100 \\ China
}

